\title{
Interpreter Provision and Hospital-Associated Outcomes Within the Limited English Proficiency Population: Analysis of Administrative Data
}

\author{
Nicole Blay, PhD 1,2, Marika Seremetkoska, GradDip ClinHons ${ }^{3}$, Jenny Morris, MCIPrac ${ }^{3}$, \\ Gael Holters, GCertDiabEduc4 ${ }^{4}$, Sharelle loannou, GradCertHlthServMgmt, GradCertCancerNg ${ }^{3}$, \\ Verily Thomas, PGDip/ntenCare/Cardiol', and Bronwyn Everett, PhD ${ }^{1,2}$
}

\begin{abstract}
${ }^{1}$ Centre for Applied Nursing Research, SWSLHD, Ingham Institute for Applied Medical Research, Liverpool, NSW, Australia; ${ }^{2}$ School of Nursing \& Midwifery, University of Western Sydney, Penrith, NSW, Australia; ${ }^{3}$ Bankstown-Lidcombe Hospital \& SWSLHD, Bankstown, NSW, Australia; ${ }^{4}$ St. George Hospital \& SESLHD, Kogarah, NSW, Australia.
\end{abstract}

$\mathrm{J}$ Gen Intern Med 34(6):820-2

DOI: $10.1007 / \mathrm{s} 11606-019-04852-8$

(c) Society of General Internal Medicine 2019

\section{INTRODUCTION}

Patients with limited English proficiency (LEP) experience longer hospital length of stay (LOS) and higher readmission rates compared with the English-speaking community. ${ }^{1}$ The impact of interpreter use on hospital outcomes, within the LEP population, is largely unknown.

Reported barriers to interpreter use include practitioners' time constraints, complexity of the interpreter booking system, and convenient access to informal interpreters. ${ }^{2}$ In this culturally diverse region in Sydney, Australia, booking the free-ofcharge interpreter service requires 3-weeks' advance notice although a 24-h emergency telephone service is available. ${ }^{3}$ The use of informal interpreters is considered a breach of policy. ${ }^{4}$

\section{METHOD}

A retrospective analysis of 19,627 de-identified and merged demographic, overnight-stay inpatient and interpreter records from one hospital for the 2014-2015 financial year were examined to determine the impact of interpreter provision on LOS and 28-day hospital readmission rates. LEP patients identified at hospital admission as needing an interpreter $(n=3074)$ were extracted and analysed (Fig. 1).

Pearson's chi-square was used for categorical values and Student's $t$ test to compare LOS and readmissions between LEP patients who received the interpreter and those who did not. A Pearson's product-moment correlation was used to compute the relationship between the frequency of interpreter provisions and LOS. Statistical analyses were performed in SPSS v23 with the significance level set at 0.05 . Ethics approval was gained for this study.

Published online February 6, 2019

\section{RESULTS}

LEP patients who received the interpreter service $(n=526)$ spoke 38 different languages and were significantly more likely to be female $(68.6 \%, n=361, p<.001)$ and younger $(t$ $(3072)=-3.79, p<.001)$ than those who did not receive the service. Interpreters were primarily provided for Arabic $(n=$ 188, 35.7\%), Vietnamese $(n=126,24.1 \%)$, Greek $(n=30$, $5.7 \%)$, Mandarin $(n=30,5.7 \%)$, and Cantonese-speaking patients $(n=30,5.7 \%)$.

Interpreter appointments were made $(n=567,18.4 \%)$ and provided for $17.1 \%(n=526)$ of LEP patients. Rescheduling of appointments $(18.9 \%, n=148)$ and cancellations $(11.9 \%$, $n=93)$ were frequent, while $7.7 \%(n=60)$ of appointments resulted in the patient, family member, or practitioner being absent at the scheduled time (reported as 'no-show'). The frequency of interpreter no-shows is not known.

LEP patients provided with an interpreter had longer LOS compared with those who did not receive the service (13.5 vs. 4.8 days, $p<.001)$. No differences between 28 -day readmission rates were found (Table 1). There was a significant correlation between the number of provided interpreter sessions and $\operatorname{LOS}(r=.55, n=3074, p=<.001)$.

\section{DISCUSSION}

Interpreter provision and the younger female demographic are explained by a higher incidence of interpreter provision to maternity patients ${ }^{5}$, and the regions' higher fertility rate compared with state average. ${ }^{6}$ The healthcare interpreter service employs interpreters speaking many languages; however, it is surmised that the spectrum of languages spoken by patients in the one hospital could pose a service challenge that possibly contributes to the low rate of interpreter provision. For practitioners, the need to book the interpreter service by telephone or provide several weeks' notice is unrealistic. Finding a mutually agreeable time between the interpreter, practitioner, patient, and/or family member(s) requires negotiation and possibly explains why $31 \%$ of interpreter appointments were 
Merged health \& interpreter datasets $(19,627$ records $(14,977$ individuals $)$

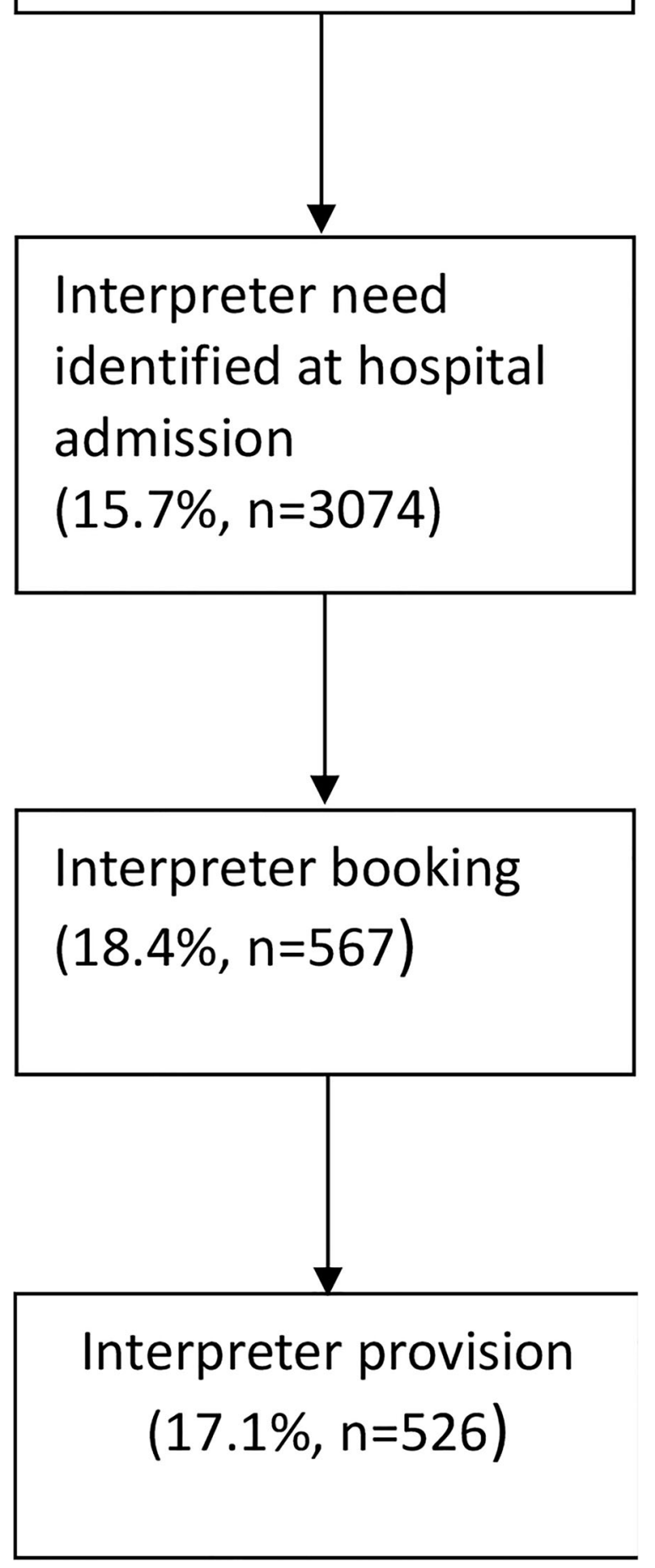

Figure 1 Flow chart of the method.
Table 1 Interpreter Service Provision and Hospital-Associated Outcomes

\begin{tabular}{|c|c|c|c|c|}
\hline \multicolumn{5}{|c|}{ Hospital-associated outcomes (days) } \\
\hline & $N(\%)$ & Mean (SD) & $t$ & $P$ value* \\
\hline \multicolumn{5}{|c|}{ Length of stay $(N=3074)$} \\
\hline $\begin{array}{l}\text { Interpreter } \\
\text { service provision }\end{array}$ & $526(17.1)$ & $13.5(18.57)$ & 18.26 & $<.001$ \\
\hline $\begin{array}{l}\text { Interpreter } \\
\text { service not } \\
\text { provided }\end{array}$ & $2548(82.9)$ & $4.8(7.07)$ & & \\
\hline \multicolumn{5}{|c|}{ Readmission $(N=829)$} \\
\hline Interpreter & $123(14.8)$ & $1.4(.50)$ & - & 0.108 \\
\hline $\begin{array}{l}\text { service provision } \\
\text { Interpreter } \\
\text { service not } \\
\text { provided }\end{array}$ & $706(85.2)$ & $1.5(.50)$ & & \\
\hline
\end{tabular}

rescheduled or cancelled. With a LOS of less than 5 days, limited time is available. It is therefore quite possible that a proportion of cancellations and 'no-shows' arose from patients undergoing a procedure or having been discharged from hospital.

LEP patients who received an interpreter remained in hospital almost three times longer than those who did not receive the service and made no impact on the 28-day readmission rate. Interpreter booking issues may influence practitioners to limit bookings for complex patients expected to remain in hospital for a longer period. Selecting patients in this way would help explain why the frequency of interpreter provisions correlated to length of stay.

The retrospective nature of this study and the use of data from independent sources limited the capacity of the researchers to verify data accuracy. Some interpreter service provisions may not have been captured or were provided following hospital discharge.

Criticism for low interpreter utilisation appears to be directed towards practitioners. To encourage compliance with policy; work-time and access issues need to be addressed. A realtime electronic interpreter booking and confirmation system may go some way to encourage service utilisation and increase efficiency.

Acknowledgments: Sincere appreciation is extended to Ms. Rachel Langdon, Ms. Katina Varelis, and Ms. Louise Smith for assistance with data provision.

Corresponding Author: Nicole Blay, PhD; Centre for Applied Nursing Research, SWSLHD Ingham Institute for Applied Medical Research, Locked Bag 7103, Liverpool, NSW 1871, Australia (e-mail: Nicole. Blay@westernsydney.edu.au).

Funding Sources This study was supported by NSW Health Nursing and Midwifery Office Strategy Reserve Funding 2015-2016.

\section{Compliance with Ethical Standards:}

Ethics approval was gained for this study. 
Conflict of Interest: BE was awarded a Sanofi-Aventis Honoraria (2016) and is a representative on the Dyslipidaemia in Nursing Advisory Board. SI was awarded funding from NSW Health Nursing and Midwifery Office Strategy Reserve Funding 2015-2016. GH is the manager for Diabetes Headquarters, Australia and New Zealand. All remaining authors declare they do not have a conflict of interest.

Publisher's Note: Springer Nature remains neutral with regard to jurisdictional claims in published maps and institutional affiliations.

\section{REFERENCES}

1. Karliner LS, Auerbach A, Nápoles A, Schillinger D, Nickleach D, PérezStable EJ. Language Barriers and Understanding of Hospital Discharge Instructions. Med Care. 2012;50:283-89.
2. Kale E, Syed HR. Language barriers and the use of interpreters in the public health services. A questionnaire-based survey. Patient Educ Couns. 2010; 81:187-91.

3. NSW Health. Health language services. NSW Ministry of Health; 2015. Available at: https://www.swslhd.health.nsw.gov.au/services/Interpreter/ bookings.html. Accessed 22 Oct 2018.

4. NSW Health. Interpreters-standard procedures for working with health care interpreters. NSW Government. Standard No.: PD2017_044.

5. Blay N, Ioannou S, Seremetkoska $\mathbf{M}$, et al. Healthcare interpreter utilisation: analysis of health administrative data. BMC Health Serv Res. 2018. https://doi.org/10.1186/s12913-018-3135-5. Accessed 22 October 2018.

6. South Western Sydney Local Health District. South Western Sydney Local Health District Year in Review 2015/16. Liverpool: SWSLHD; 2016. 